\title{
Design of Manipulator Control System of Forest Picking Robot Based on Fractional Order PID Sliding Mode Control
}

\author{
Lei Shi ${ }^{1,2}$, Miao Dang ${ }^{1,2}$ \\ ${ }^{1}$ Henan Polytechnic Institute, Nanyang, Henan 473000, China \\ ${ }^{2}$ Henan industrial robot application engineering technology research center, Nanyang, Henan, China \\ *Corresponding Author.
}

\section{Abstract:}

This paper studies the control system design of forest picking robot manipulator based on fractional PID sliding mode control. In this paper, the structure characteristics, learning algorithm and application of fractional order PID sliding mode control in manipulator control are analyzed. In this paper, the nonlinear approximation property of fractional order PID sliding mode control is theoretically verified. This paper analyzes the basic structure of picking manipulator system in detail. At the same time, the Lagrange Euler method is used to deduce the dynamic equation of the two degree of freedom series manipulator, and the inertia characteristics, Coriolis force and centripetal force characteristics, heavy torque characteristics are analyzed. The nonlinear system model of manipulator based on S-function is established in MATLAB, and the dynamic model is transformed into the form of second-order differential equation to facilitate the introduction of the designed algorithm.

Keywords: Forest, Forestry Picking, PID Synovial Control, Manipulator

\section{INTRODUCTION}

The controller designed in this paper belongs to open controller. With the development of robot technology and the continuous expansion of robot application field, higher requirements are put forward for the performance of robot [1-2]. Therefore, how to effectively apply the research results of other fields (such as image processing, sound recognition, optimal control, artificial intelligence, etc.) to the real-time operation of robot control system is a challenging research work [3]. The research of open robot controller is undoubtedly of great significance to improve the performance and autonomy of robot and promote the development of robot technology. 
Article History: Received: 28 October 2021 Revised: 05 December 2021 Accepted: 10 January 2022 Publication: 28 February 2022

The main content of this paper is the design of 6-DOF fruit picking manipulator control system, which is a part of the overall manipulator project design [4-5]. The overall experimental project consists of three parts: fruit image recognition, camera and fruit positioning, and the design of manipulator control system [6]. The six degree of freedom fruit picking manipulator gives the spatial coordinates of the fruit through the fruit recognition and visual positioning system, and determines the rotation angle of the steering gear after the corresponding conversion between the spatial coordinates and the steering gear angle [7]. The angle of the steering gear is controlled by the control system, and then the posture of the manipulator is changed to realize the purpose of accurate fruit picking [8=9]. The application of picking manipulator can solve the problem of insufficient agricultural labor force: replace human beings to complete dirty or very hard labor; It can replace people in mechanical monotonous work; Agricultural products with high quality and added value can be obtained; Realize aseptic production in the factory; It can attract more young scholars to carry out agricultural production $\mathrm{R} \& \mathrm{D}$ [10]. Therefore, fruit picking robot will play a powerful role in future agricultural production.

\section{RESEARCH ON CONTROL METHOD OF MANIPULATOR}

\section{Working principle of micro servo DC motor}

The working principle of micro servo motor is a relatively simple process, because a single micro servo DC motor is a typical closed-loop feedback system, and its principle is shown in Figure 1.

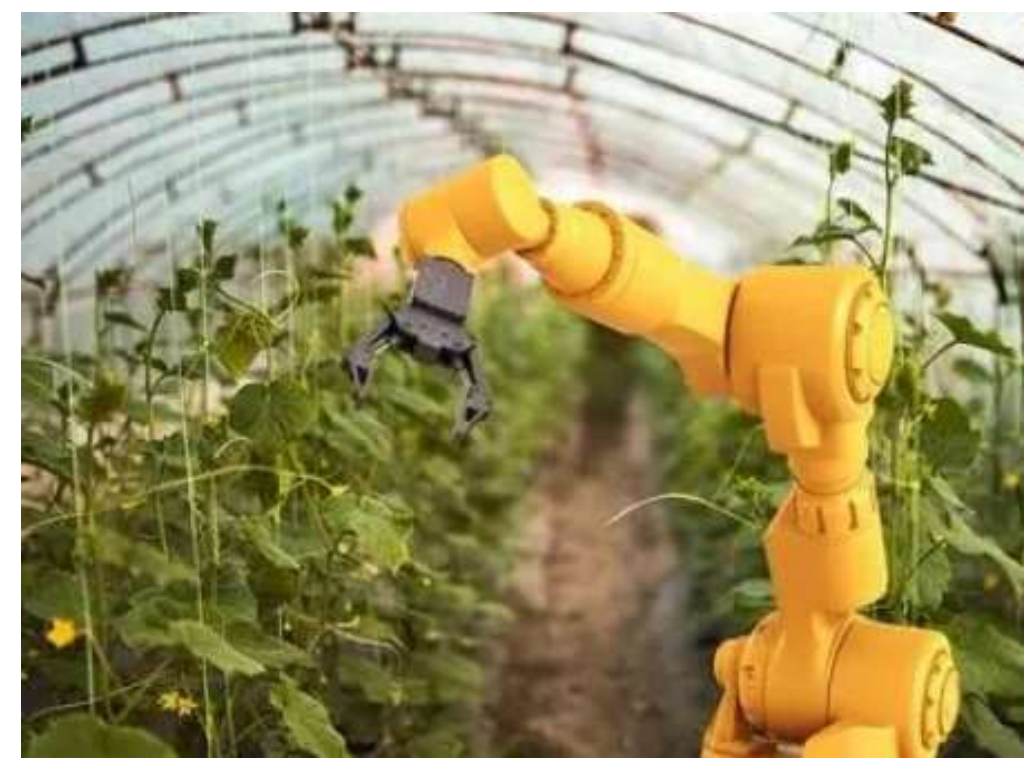


Fig 1: Working principle diagram of micro servo DC motor

The working principle diagram shown in Figure 1 is explained: the control pulse is transmitted to the DC motor part through the control circuit, and then the variable speed gear set is driven by the motor. The terminal (output end) drives a linear proportional potentiometer for position detection. The potentiometer converts the angle coordinate into a proportional voltage and feeds it back to the control circuit board. The control circuit board compares it with the input control pulse signal to generate a correction pulse. And drive the motor to rotate forward or reverse, so that the output position of the gear set is consistent with the expected value, and the correction pulse tends to 0 , so as to achieve the purpose of accurate positioning of the servo motor. For example, when a periodic forward pulse signal is input, its high-level time lasts for $1 \mathrm{~ms}-2 \mathrm{~ms}$ and low-level time lasts for $5 \mathrm{~ms}-20 \mathrm{~ms}$.

According to the analysis of the above pulse signal diagram, the variable speed principle is based on PWM pulse width. The control signal enters the signal modulation chip from the channel of the receiver to obtain the DC bias voltage. There is a reference circuit inside it, which generates a reference signal with a period of $20 \mathrm{~ms}$ and a width of $1.5 \mathrm{~ms}$. The obtained DC bias voltage is compared with the voltage of the potentiometer to obtain the voltage difference output. Finally, the positive and negative voltage difference is output to the motor drive chip to determine the forward and reverse rotation of the motor. When the motor speed is constant, the potentiometer is driven to rotate through the cascade reduction gear, so that the voltage difference is 0 and the motor stops rotating. That is, when the input pulse width is less than $1.5 \mathrm{~ms}$, the motor rotates counterclockwise, when the input pulse width is equal to $1.5 \mathrm{~ms}$, the motor stops, and when the pulse width is greater than $1.5 \mathrm{~ms}$, the motor rotates clockwise.

\section{Internal structure of micro servo DC motor}

Micro servo DC motor, also known as steering gear, was mainly used for model aircraft control in the early stage. With the rapid development of robot research in recent years, steering gear is more and more used in the field of robot. In recent years, the technology of steering gear has developed very rapidly. Smaller volume, higher speed and greater torque are the development direction of steering gear. The steering gear has the advantages of simple control, large output torque, accurate output angle, low working voltage and standard modularization. It is very suitable for the manufacture of small robots.

The physical principle of the steering gear is that the receiver sends a signal to the steering gear, judges the rotation direction through the circuit board, drives the coreless motor to start rotation, transmits the power to the swing arm through the reduction gear, and judges whether the positioning has been reached by the feedback signal of the position detector. The position detector is essentially a variable resistance. When the steering gear rotates, the resistance value will change accordingly. The rotation angle can be known by detecting the resistance value. The general servo motor winds the thin copper wire on the three pole rotor. When the current flows 
Article History: Received: 28 October 2021 Revised: 05 December 2021 Accepted: 10 January 2022 Publication: 28 February 2022

through the coil, it will produce a magnetic field, repel the magnet around the rotor, and then produce a rotating force. According to the principles of physics, the moment of inertia of an object is directly proportional to its mass. Therefore, the greater the mass, the greater the force required to rotate the object. In order to achieve fast speed and low power consumption, the steering gear winds the thin copper wire into a very thin hollow cylinder to form a very light five pole hollow rotor, and places the magnet in the cylinder, which is a coreless motor.

The standard micro servo DC motor has three control lines: power line, control line and ground wire. The signal line is generally white or yellow, the positive power line is red and the negative power line is black. Do not connect the circuit reversely here, otherwise the control chip is easy to burn out. The power line and ground wire provide energy for the motor and control line. The voltage is $4 \mathrm{v}-6 \mathrm{v}$. This voltage should be isolated from the power supply of the processor as far as possible (because the servo motor will produce some noise and interference). When the servo motor exceeds the rated load, it will also pull down the voltage of the amplifier, so the power supply proportion of the system must be reasonable.

\section{HARDWARE DESIGN OF SIX DEGREE OF FREEDOM FRUIT PICKING MANIPULATOR CONTROL SYSTEM}

1.Hardware development tools and PCB design methods

The EDA software in the design adopts Altium designer 08 of Altium company. Compared with altiumdesigner 6.0, altiumdesigner 08 has brought some meaningful new functions and enhancements, which makes design management easier and expands FPGA design. It is a new version of the fully integrated electronic product development system. It is also the first and only complete board level design solution in the industry. Aitium designer 6.0 not only fully inherits the functions and advantages of previous versions including 99SE and Protel 2004, but also adds many improvements and high-end functions.

PCB wiring principle: in PCB design, wiring is an important step to complete product design. In the whole PCB, the design process of wiring is the highest, the skills are the smallest and the workload is the largest. PCB wiring includes single-sided wiring, double-sided wiring and multi-layer wiring. There are also two ways of wiring: automatic wiring and interactive wiring. Before automatic wiring, the lines with strict requirements can be wired interactively in advance. The side lines of input and output terminals should avoid adjacent parallel to avoid reflection interference.

Treatment of power supply and ground wire: the wiring of power supply and ground wire shall be taken seriously to minimize the noise interference generated by power supply and ground wire, so as to ensure the quality of products. Generally, a coupling capacitor is added between the power supply and ground wire to widen the width of the power supply and ground wire as much as possible. It is better that the ground wire is wider than the power line width. 
Article History: Received: 28 October 2021 Revised: 05 December 2021 Accepted: 10 January 2022 Publication: 28 February 2022

Their relationship is: ground wire > power wire > signal wire.

Design rule check (DRC): after the wiring design is completed, it is necessary to carefully check whether the wiring design meets the rules formulated by the designer, and also confirm whether the rules formulated meet the requirements of printed board production process.

\section{Power module}

In this experiment, the power module provides the required voltage for the 6-DOF manipulator and the arm control board. The design of the power circuit is shown in Figure 2. In order to be simple and convenient, a double pin power supply circuit is designed, the power supply is controlled by switching so on, and a light-emitting diode is designed to monitor the power supply. The $1 \mathrm{~K}$ resistor protects the LED and leads to two double pin power interfaces, which is convenient to provide power for other peripheral module circuits.

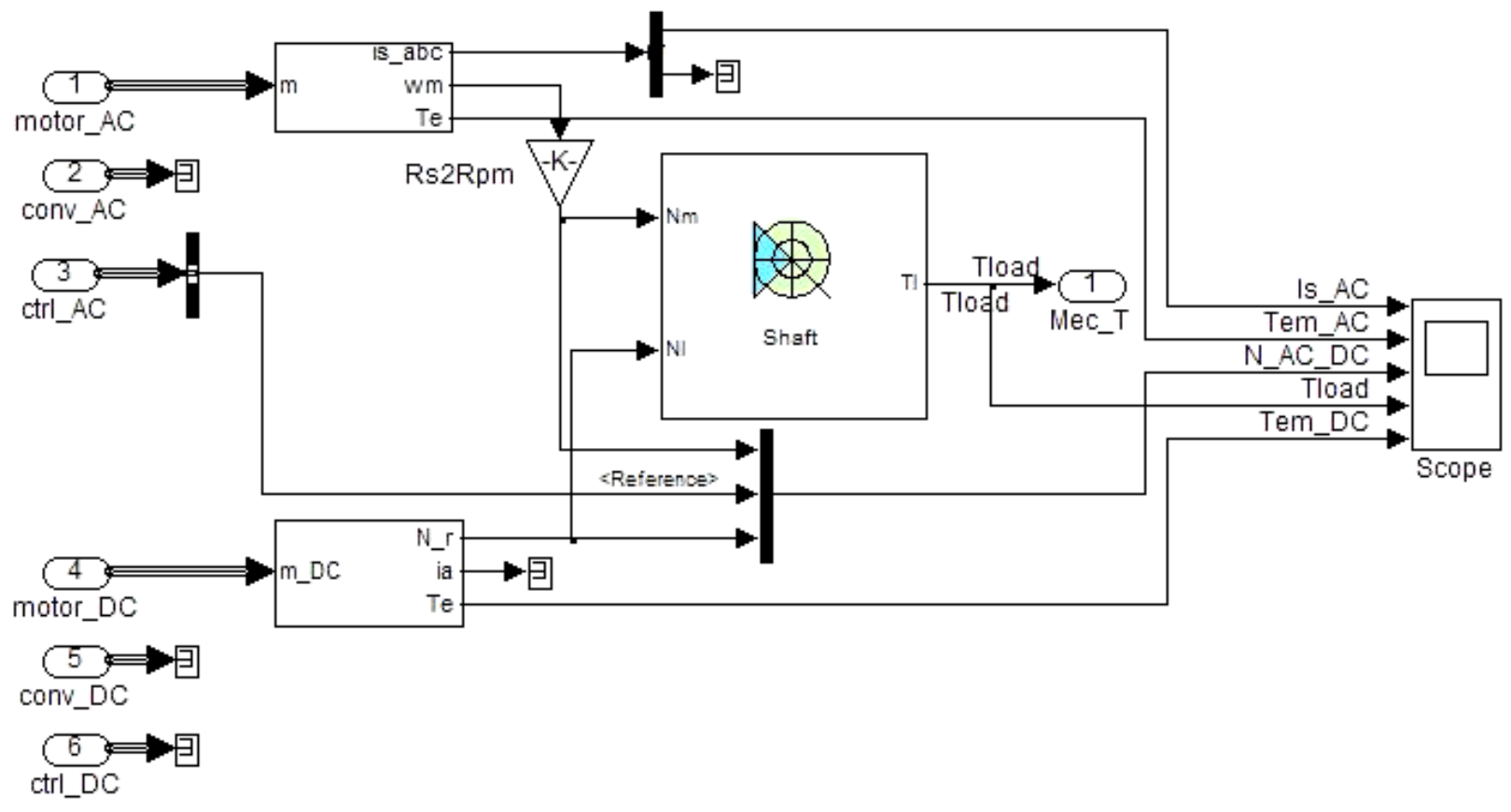

Fig 2: Schematic diagram of power supply circuit

3.Keyboard command input module.

To realize the control of the six-degree-of-freedom manipulator, it is necessary to operate the manipulator with multiple keys. Therefore, the design of the hardware circuit board adopts the control mode of $4 \times 4$ matrix keyboard to input commands to realize the control of the steering gear of the six-degree-of-freedom fruit picking manipulator. Touch switch with 4 pins is adopted, and its specification is $6.0 \times 6.0 \times 5.0 \mathrm{~mm}$. It is an electronic switch, which can be turned on by gently pressing the switch button when in use, and can be turned off when the 
Article History: Received: 28 October 2021 Revised: 05 December 2021 Accepted: 10 January 2022 Publication: 28 February 2022

hand is released, and its internal structure can be turned on and off by the elastic force of metal shrapnel. When in use, two feet far away can be shorted, and the four corners are for more stable welding. The design of this circuit board uses two pins in the lower row of keys, and the matrix keyboard module interface is designed with pins, which facilitates the expansion of system functions.

4.Steering gear interface module

The essence of the control of the 6-DOF fruit picking manipulator is to control the steering gear of each joint of the manipulator. The manipulator is composed of six actuators. Therefore, the actuator interface module is the core to realize the communication control between the chip module and the whole six degree of freedom fruit picking manipulator.

TABLE I. Pin description of JM12864M

\begin{tabular}{|c|c|c|c|}
\hline PIN & $\begin{array}{c}\text { PIN } \\
\text { NAME }\end{array}$ & DIRECTION & FUNCTION DESCRIPTION \\
\hline 1 & VSS & - & Power ground of the module \\
\hline 2 & VDD & $+5 \mathrm{v}$ & Positive power terminal of the module \\
\hline 3 & V0 & - & LCD drive voltage input \\
\hline 4 & RS(CS) & H/L & $\begin{array}{c}\text { Parallel instruction "according to selection signal: serial chip } \\
\text { selection signal }\end{array}$ \\
\hline 5 & RlW(SID) & H/L & Parallel read / write selection signal; Serial data port \\
\hline 6 & E(CLK) & H/L & Parallel enable signal, serial synchronous clock \\
\hline 7 & DB0 & H/L & Data 0 \\
\hline 8 & DB1 & H/L & Data 1 \\
\hline 9 & DB2 & H/L & Data 2 \\
\hline 10 & DB3 & H/L & Data 3 \\
\hline 11 & DB4 & H/L & Data 4 \\
\hline 12 & DB5 & H/L & Data 5 \\
\hline 13 & DB6 & H/L & Data 7 \\
\hline 14 & DB7 & H/L & Empty foot \\
\hline 15 & PSB & H/L & Parallel / serial interface selection: H-parallel; L-serial \\
\hline 16 & NC & & Reset, low level active \\
\hline 17 & RESET & H/L & Empty foot \\
\hline 18 & NC & & Backlight cathode \\
\hline 19 & LED_A & $($ LED+5v) & Backlight cathode \\
\hline 20 & LED_K & $($ LED+5v) & \\
\hline & & &
\end{tabular}




\section{Software design of 6-DOF fruit picking manipulator control system}

ATMEL AVR Studio integrated development environment (IDE), including AVR Assembler compiler, AVR Studio debugger, and supporting chip download programming functions. AVR Studio supports the following development tools: AVRISPMKII, JTAGICE MKII, AVRDragon, JTAGICE, AVRISP, stk500/501/502/503/504/505/520, ICES0, ICE40 and ICE200. The main functions of AVR Studio include the following: compiling and compiling assembly projects; Use STK500, JTAG or JTAGMKII to download; Debugging can be done by software simulation, JTAG simulation or JTAG MKII simulation.

The main program is the soul of the whole six degree of freedom fruit picking manipulator control system. The main program can organically call each subroutine, make them form a closely connected whole, and control each module to execute operation commands in an orderly manner. The overall design flow of the main program includes system initialization, keyboard detection and scanning, LCD display, steering gear control and other modules.

After the system starts, the system initialization is carried out first. The system initialization includes I / O port register initialization, 12864 LCD initial content, data and instruction initialization, keyboard detection and scanning initialization, etc. After initialization, scan the keyboard to judge whether a key is pressed. If yes, delay de chattering and re judge. To ensure that a key is pressed, then find the close key and wait for the key to be released, and then determine the key code. Next, enter the timer interrupt. At this time, the LCD screen displays the angle state of the steering gear in real time. After the operation process, return to the keyboard scan and wait for the input of the next instruction.

In the experiment, the six-degree-of-freedom fruit picking manipulator was controlled by matrix keyboard. In order to realize the optimal design of the controller of the six-degree-offreedom fruit picking manipulator, it is necessary to understand the detection principle and design the software program. The matrix keyboard detection adopts the line scanning method, also known as the line scanning query method. Is one of the most commonly used key recognition methods.

In the control experiment of the 6-DOF fruit picking manipulator, the LCD screen -JM12864 is used to display the status of the steering gear in real time, so as to ensure the accuracy of the angle input of the steering gear of the manipulator. Liquid crystal display principle: dot matrix liquid crystal display module (LCM) jml2864 is a 128 column and 64 row array composed of 128 X64 liquid crystal display points. Each display point corresponds to a binary number. 1 means on and 0 means off. The ram that stores these lattice information is called the display data memory. To display a graphic or Chinese character is to write the corresponding dot matrix information into the corresponding storage unit.

\section{CONCLUSION}


Agricultural picking robot is a hot research topic at home and abroad. It integrates many disciplines such as agricultural information, machinery, electronics, sensors, materials, control theory, computer, information processing and so on. The research of agricultural picking robot is undoubtedly of great practical significance to promote the development of science and technology and human process. The combination of efficient microcontroller and robot technology is an inevitable trend of today's scientific and technological development, which provides a new way for the development of agricultural modern electromechanical products. In particular, the development of agricultural robot technology often represents the level of agricultural automation in a country. The 6-DOF fruit picking manipulator studied in this paper is a multi joint robot based on the combination of agricultural application and electromechanical technology to realize specific functions. The manipulator can be used as a learning platform for demonstration and demonstration, and can also be applied to the research of agricultural picking robot after adding corresponding detection sensors and cutting devices.

\section{ACKNOWLEDGEMENTS}

This research was supported by the Promotion Special Project of the Scientific Study Program of Henan Province (Grant No. 202102210084).

\section{REFERENCES}

[1]Zhang, Tiezhong, and B. Lin. "Object extraction for the vision system of fruit picking robot." Journal of China Agricultural University 9.2(2004):68-72.

[2]Gu, B., et al. "Design and experiment of intelligent mobile fruit picking robot." Nongye Jixie Xuebao/transactions of the Chinese Society of Agricultural Machinery 43.6(2012):153-160.

[3]Wei, Xiangqin, et al. "Automatic method of fruit object extraction under complex agricultural background for vision system of fruit picking robot." Optik - International Journal for Light and Electron Optics 125.19(2014):5684-5689.

[4]Fu, Xiao Jin, et al. "The Fruit-Picking Robot." Applied Mechanics \& Materials 610(2014):515-518.

[5]Chiu, Yi Chich, S. Chen, and J. F. Lin. "Study of an Autonomous Fruit Picking Robot System in Greenhouses *." Engineering in Agriculture Environment \& Food 6.3(2013):92-98.

[6]Ji, Wei, et al. "Compliance grasp force control for end-effector of fruit-vegetable picking robot." Transactions of the Chinese Society of Agricultural Engineering 30.9(2014):19-26.

[7]Zhao, Dong Hui, H. Zhang, and J. X. Hou. "Design of Fruit Picking Device Based on the Automatic Control Technology." Key Engineering Materials 620(2014):471-477. 
Article History: Received: 28 October 2021 Revised: 05 December 2021 Accepted: 10 January 2022 Publication: 28 February 2022

[8]Peng, Hongxing, et al. "Fruit image segmentation based on evolutionary algorithm." Nongye Gongcheng Xuebao/transactions of the Chinese Society of Agricultural Engineering 30.18(2014):294-301.

[9]Fu, L., et al. "Development and experiment of end-effector for kiwifruit harvesting robot." Nongye Jixie Xuebao/transactions of the Chinese Society of Agricultural Machinery 46.3(2015):1-8.

[10]Schuetz, C., et al. "Evaluation of a direct optimization method for trajectory planning of a 9DOF redundant fruit-picking manipulator." International Telecoms Sync Forum Itsf 2015.5(2015):2660-2666. 